\title{
Silicone gel em cicatrizes de cirurgia plástica: estudo clínico prospectivo
}

\author{
Silicone gel in plastic surgery scars: a prospective study
}

\author{
Henrique N. Radwanski ${ }^{1}$ \\ WANDA ElizABETH \\ MASSIERE Y CORREA ${ }^{2}$ \\ Tiago José Refosco ${ }^{3}$ \\ AdILSON FARRAPEIRA JUNIOR ${ }^{4}$ \\ Ivo PitanguY ${ }^{5}$
}

Trabalho realizado no Instituto Ivo Pitanguy, $38^{\mathrm{a}}$ Enfermaria da Santa Casa da Misericórdia, Rio de Janeiro, RJ, Brasil.

Artigo submetido pelo SGP (Sistema de Gestão de Publicações) da RBCP.

Artigo recebido: $11 / 7 / 2010$ Artigo aceito: $24 / 8 / 2010$

\begin{abstract}
RESUMO
Introdução: O uso de gel de silicone em cicatrizes data do começo da década de 1980. Desde então, um grande número de trabalhos científicos, e dezenas de diferentes fórmulas do produto, vêm sendo publicados e experimentados, atestando os benefícios do gel de silicone na prevenção de cicatrizes hipertróficas e quelóides. Apesar do mecanismo exato de ação do silicone ainda ser desconhecido, a hipótese mais aceita é que o curativo oclusivo estimula os queratinócitos à maior secreção de fatores de crescimento localmente, influenciando consequentemente a regulação dos fibroblastos. Método: Com o objetivo de comprovar os benefícios do silicone gel na melhora clínica das cicatrizes de etiologia cirúrgica eletiva, foi realizado um estudo prospectivo, numa população miscigenada, no Serviço de Cirurgia Plástica na $38^{\mathrm{a}}$ Enfermaria da Santa Casa da Misericórdia do Rio de Janeiro. Resultados: O gel de silicone demonstrou ação favorável em cicatrizes pós-cirurgia plástica. Conclusão: O uso de silicone gel mostrou-se útil na melhora de cicatrizes recentes, melhorando parâmetros subjetivos e objetivos, como eritema, prurido e endurecimento.
\end{abstract}

Descritores: Cirurgia plástica. Géis de silicone. Cicatrização de feridas.

\section{SUMMARY}

Background: The use of silicone gel on surgical wounds began in the 1980's. Since then, a large number of scientific papers and dozens of different formulas have been published and experimented, attesting the benefits of this product in the prevention of hypertrophic scars and keloids. Although the exact mechanism of action of silicone gel has not yet been elucidated, the most widely accepted theory explains that the occlusive film stimulates the keratinocytes to increase the local secretion of growth factors, subsequently influencing the regulation of fibroblasts. Methods: A prospective study was undertaken at the $38^{\text {th }}$ Ward of the Santa Casa General Hospital, Rio de Janeiro, to evaluate the positive effects of silicone gel on surgical wounds, in a mixed race population. Results: Silicone gel demonstrated a positive effect on surgical wounds following plastic surgery. Conclusion: Silicone gel is useful to enhance the quality of recent scars, in both subjective and objective parameters, such as erythema, pruritus and firmness.

Descriptors: Plastic surgery. Silicone gels. Wound healing.

1. Membro titular da Sociedade Brasileira de Cirurgia Plástica (SBCP); Professor assistente e assessor científico do Instituto Ivo Pitanguy.

2. Membro titular da SBCP; Professor assistente, Coordenadora Geral de Ensino e Pesquisa do Instituto Ivo Pitanguy.

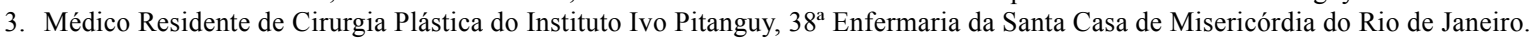

4. Membro associado da SBCP.

5. Membro titular e patrono da SBCP; Professor titular, Pontifícia Universidade Católica do Rio de Janeiro e Instituto de Pós-Graduação Médica Carlos Chagas. Presidente honorário do Instituto Ivo Pitanguy. 


\section{INTRODUÇÃO}

Melhorar o aspecto final de uma cicatriz é um desafio que interessa às diversas especialidades médicas, em especial à cirurgia plástica. A evolução natural de uma incisão cutânea resulta, na maioria dos casos, numa cicatriz linear e fina, mas sempre existe o risco de uma cicatriz hipertrófica ou da formação de um quelóide ${ }^{1,2}$. Apesar da evolução do conhecimento do processo de cicatrização, ainda não é possível realizar uma incisão cutânea sem deixar uma cicatriz, pois ela representa a consequência de todo ato cirúrgico. $\mathrm{O}$ cirurgião plástico deve, portanto, planejar a extensão e a localização da sua incisão, considerando a possibilidade de haver uma cicatriz de má qualidade.

O silicone, nas mais diversas formulações, tem sido usado na prevenção de cicatrizes hipertróficas ou quelóides, desde os anos 1980, quando artigos científicos demonstraram o benefício destes produtos em cicatrizes consequentes de incisões cirúrgicas, áreas de queimadura e trauma, entre outros. $\mathrm{O}$ resultado da grande maioria dos trabalhos aponta para melhora significativa na qualidade final das cicatrizes, de diferentes etiologias, após o uso de produtos à base de silicone gel ${ }^{3-8}$. É interessante notar, entretanto, que a totalidade dos trabalhos foi realizada em pacientes de pele clara. Sabe-se que existe maior tendência à cicatrização hipertrófica ou quelóide nas pessoas de pele mais escura.

Este trabalho teve como interesse avaliar a evolução das cicatrizes em uma população miscigenada, usando um produto contendo silicone gel de rápida secagem, em cicatrizes exclusivamente resultantes de cirurgia plástica. Assim, foi planejado um estudo prospectivo, no Serviço de Cirurgia Plástica do Prof. Ivo Pitanguy, na $38^{\text {a }}$ Enfermaria da Santa Casa da Misericórdia do Rio de Janeiro.

\section{MÉTODO}

\section{Casuística}

Os pacientes estavam matriculados na $38^{\mathrm{a}}$ Enfermaria da Santa Casa da Misericórdia do Rio de Janeiro / Instituto Ivo Pitanguy e foram selecionados de acordo com critérios de inclusão e exclusão do estudo. Os critérios de inclusão considerados foram: idade entre 15 e 65 anos, cicatriz cirúrgica recente e assinatura do termo de consentimento livre e esclarecido. Os critérios de exclusão considerados foram: presença de doença sistêmica ou metabólica ou de ferida cirúrgica aberta, paciente grávida ou com potencial de engravidar, uso de outro medicamento concomitante na cicatriz, participação em outro estudo clínico em andamento ou nos 60 dias precedentes, antecedentes de alergia ao silicone e incapacidade de aplicação do gel.

O estudo foi realizado entre novembro de 2007 e abril de 2009, num total de 128 pacientes consecutivos. Foram avaliados somente pacientes que se encontravam em pósoperatório de cirurgia plástica, e que tinham indicação de tratar cicatrizes na tentativa de obter um melhor resultado estético.

Dentre os pacientes que finalizaram o estudo, $96 \%$ tinham a origem das cicatrizes em cirurgias realizadas no próprio serviço, e $4 \%$, originados de outro serviço.

O estudo foi aprovado pelo Comitê de Ética em Pesquisa da Santa Casa da Misericórdia do Rio de Janeiro e foi realizado de acordo com a Declaração de Helsinki, revisada em 2000, e a Resolução 196/96 do Conselho Nacional de Saúde.

\section{Parâmetros de avaliação}

Todos os pacientes selecionados foram submetidos ao tratamento da cicatriz cirúrgica na fase de remodelação (entre a retirada das últimas suturas e o $3^{\circ}$ mês de pós-operatório), exclusivamente com silicone gel (Polysiloxanes, Silicon Dioxide, Kelo-cote ${ }^{\circledR}$, Farmoquímica) em toda extensão da cicatriz, duas vezes ao dia, num período de 3 a 6 meses. Os pacientes foram avaliados em três períodos distintos da evolução. A primeira avaliação se deu um mês após o início do tratamento, a segunda, no segundo ao quinto mês de uso do produto e a avaliação final, entre o terceiro e sexto mês de uso do produto. Na avaliação inicial, foi realizada a anamnese, coleta de dados demográficos, e verificação dos critérios para inclusão e exclusão no estudo.

Em todas as avaliações, os pacientes responderam ao questionário de qualidade de vida e suas cicatrizes foram avaliadas por um dos médicos pesquisadores.

A avaliação de eficácia do tratamento foi realizada por meio da escala de Vancouver (Quadro 1).

Cabe também ressaltar que foi realizada documentação fotográfica desde a avaliação inicial até o momento da alta clínica de todos os pacientes que concluíram o estudo.

\section{Análise estatística}

A análise estatística foi composta dos seguintes parâmetros:

- Variação na escala de Vancouver ao longo de três momentos de avaliação (inicial, intermediário e final), analisada pela ANOVA de Friedman e seu respectivo teste de comparações múltiplas;

- Variação de dados categóricos (eritema, relevo e flexibilidade) ao longo de três momentos de avaliação foi analisada pelo teste de Mc Nemar corrigido.

Dados paramétricos serão apresentados como média \pm desvio padrão e os não-paramétricos, como mediana. Foram utilizados métodos não-paramétricos, pois as variáveis não apresentaram distribuição normal devido à dispersão dos dados e a falta de simetria da distribuição. O critério de determinação de significância adotado foi o nível de 5\%. A análise estatística foi processada pelo software estatístico SAS system ${ }^{\circledR}$. 


\section{Quadro 1. Evolução - Escala de Vancouver.}

\begin{tabular}{l}
\hline Eritema \\
\hline Roxo \\
\hline Vermelho \\
\hline Rosa (com alguns componentes avermelhados) \\
\hline Cor de pele normal (com alguns componentes rosa) \\
\hline Cor de pele normal \\
\hline
\end{tabular}

\section{Relevo (altura)}

\begin{tabular}{l|c}
\hline $6,0 \mathrm{~mm}$ & $6,0 \mathrm{~mm}$ \\
\hline $4,0-6,0 \mathrm{~mm}$ & $3,0-6,0 \mathrm{~mm}$ \\
\hline $2,0-4,0 \mathrm{~mm}$ & $1,0-3,0 \mathrm{~mm}$ \\
\hline $0,1-2,0 \mathrm{~mm}$ & $0,1-1,0 \mathrm{~mm}$ \\
\hline Normal/Plano & Normal/Plano \\
\hline
\end{tabular}

Flexibilidade (endurecimento)

Aderente - firme e aderente às estruturas locais que cercam a cicatriz

Firme - inflexível, difícil de mover, resistente à pressão manual

Flexível - com uma resistência moderada

Flexível - com uma resistência mínima

Normal

Prurido $0 \_5 \quad 10$

nota 0 (sem prurido) até 10 (pior prurido experimentado)

Sensibilidade (dor) $0 \_5 \_10$

nota 0 (sem dor) até 10 (pior dor experimentada)

\section{RESULTADOS}

As características demográficas da população do estudo estão apresentadas na Tabela 1 . O sexo feminino representou $98 \%$ (125 de 128) da amostra; em $80 \%$ dos pacientes não havia qualquer comorbidade.

No total, 128 pacientes foram incluídos no estudo, mas a análise longitudinal com os dados realizados em três momentos foi de 69 pacientes. Na Tabela 2, descrevemos as razões para o término do tratamento. Podemos observar que 69 concluíram o estudo de acordo com o planejado, portanto são estes os pacientes incluídos nas análises do presente estudo.

A grande maioria da perda foi atribuída à desistência por parte dos pacientes, principalmente em decorrência dos bons resultados obtidos, sobretudo no início do tratamento,
Tabela 1. Características da população do estudo.

\begin{tabular}{|c|c|c|c|}
\hline & & $\mathbf{n}$ & $\%$ \\
\hline Gênero & Feminino & 67 & 97 \\
\hline Tabagismo & $\operatorname{Sim}^{\mathrm{a}}$ & 11 & 15,9 \\
\hline \multirow{3}{*}{$\begin{array}{l}\text { Comorbidades } \\
\text { importantes }\end{array}$} & ausente & 55 & 79,7 \\
\hline & HAS & 9 & 13 \\
\hline & outras $^{\mathrm{b}}$ & 5 & 7 \\
\hline \multirow{3}{*}{$\begin{array}{l}\text { Histórico de } \\
\text { cicatrização } \\
\text { anormal }\end{array}$} & ausente & 64 & 92,8 \\
\hline & história pessoal & 4 & 5,8 \\
\hline & história familiar & 1 & 1,4 \\
\hline \multirow[t]{5}{*}{ Tipo de pele } & sempre se queima & 3 & 4,4 \\
\hline & $\begin{array}{c}\text { normalmente se } \\
\text { queima }\end{array}$ & 14 & 20,6 \\
\hline & $\begin{array}{c}\text { algumas vezes se } \\
\text { queima }\end{array}$ & 26 & 38,2 \\
\hline & nunca se queima & 25 & 36,8 \\
\hline & $\begin{array}{l}\text { Duração do tratamento } \\
\text { (meses, mediana) }\end{array}$ & 69 & 6 \\
\hline
\end{tabular}

atual + ex-tabagista

${ }^{\mathrm{b}}$ asma, rinite alérgica, hipercolesterolemia, nefrolitíase, labirintite
Tabela 2. Término do tratamento.

\begin{tabular}{l|c|c|c}
\hline Variável & Categoria & $\mathbf{n}$ & $\mathbf{\%}$ \\
\hline \multirow{4}{*}{$\begin{array}{l}\text { Motivodotérmino } \\
\text { do tratamento }\end{array}$} & $\begin{array}{c}\text { De acordo com o } \\
\text { planejamento }\end{array}$ & 69 & 53,9 \\
\cline { 2 - 4 } & $\begin{array}{c}\text { Interrupção do } \\
\text { paciente }\end{array}$ & 40 & 31,3 \\
\cline { 2 - 4 } & $\begin{array}{c}\text { Paciente não } \\
\text { disponível }\end{array}$ & 1 & 0,8 \\
\cline { 2 - 4 } & $\begin{array}{c}\text { Alergia/Efeitos } \\
\text { adversos }\end{array}$ & 5 & 3,9 \\
\cline { 2 - 4 } & Outros & 13 & 10,2 \\
\hline
\end{tabular}


favorecendo assim a descontinuidade do uso do produto, mas parte da perda é atribuída à ocorrência de eventos adversos.

Com relação a efeitos adversos, houve incidência de $6,25 \%(n=8)$ do total de pacientes, principalmente eritema e prurido cutâneo, melhorando com a descontinuação ou pausa no tratamento, não havendo necessidade de qualquer tratamento específico.

$\mathrm{Na}$ Tabela 3, estão apresentados os dados relacionados ao tipo de cicatriz tratada no estudo. A grande maioria das pacientes foi submetida a procedimentos de contorno corporal, o que justifica o fato do maior número de cicatrizes localizadas em tórax e abdome. Oitenta e seis $(67,2 \%)$ pacientes tiveram suas cicatrizes classificadas inicialmente como normais e $28(21,8 \%)$, como hipertróficas. Foi observado comprimento mediano de $475 \mathrm{~mm}$ e largura de $2,5 \mathrm{~mm}$ das cicatrizes avaliadas.

A Tabela 4 fornece a frequência (n) e o percentual (\%) dos sintomas/sinais iniciais da cicatriz na amostra de 69 pacientes. Observou-se que o sintoma mais frequente foi o prurido $(58,6 \%)$, seguido de fisgada $(26,6 \%)$ e dor $(32 \%)$; em relação aos sinais, foi o eritema $(64,8 \%)$, seguido do relevo $(28,9 \%)$.

\section{Avaliação de eficácia}

A Figura 1 mostra gráfico com somatório da pontuação na escala de Vancouver nos momentos inicial, intermediário e final.

Segundo os testes aplicados, houve melhora significativa nas cicatrizes tratadas com o produto, tomando-se como parâmetro a escala de Vancouver $(\mathrm{p}<0,0001)$ ao longo do tratamento, principalmente entre o momento inicial e intermediário (nos $1^{\text {os }}$ três meses), observada por diminuição na pontuação total.

Com relação à análise da sintomatologia em particular, houve melhora significativa no quesito prurido e na sensibilidade $(\mathrm{p}<0,0001)$ ao longo do tratamento, principalmente entre o momento inicial e intermediário (Figura 1).

Uma análise qualitativa da cicatriz foi realizada em relação a eritema, relevo e flexibilidade das cicatrizes em tratamento, sendo incluídos somente os pacientes que completaram as três revisões ( $\mathrm{n}=69)$. Nas três características analisadas, houve melhora estatisticamente significativa, porém, com relação ao relevo, a melhora importante se deu entre o momento inicial e final $(p<0,0001)$, sendo que eritema e flexibilidade mostraram resposta mais rápida, como observado com maior detalhe na Figura 2.

A eficácia do tratamento foi considerada muito boa em $62 \%(n=42)$ dos pacientes, boa em $28 \%(n=19)$, moderada em 7\% $(n=6)$ e insatisfatória em 3\% $(n=2)$.
Tabela 3. Características da cicatriz.

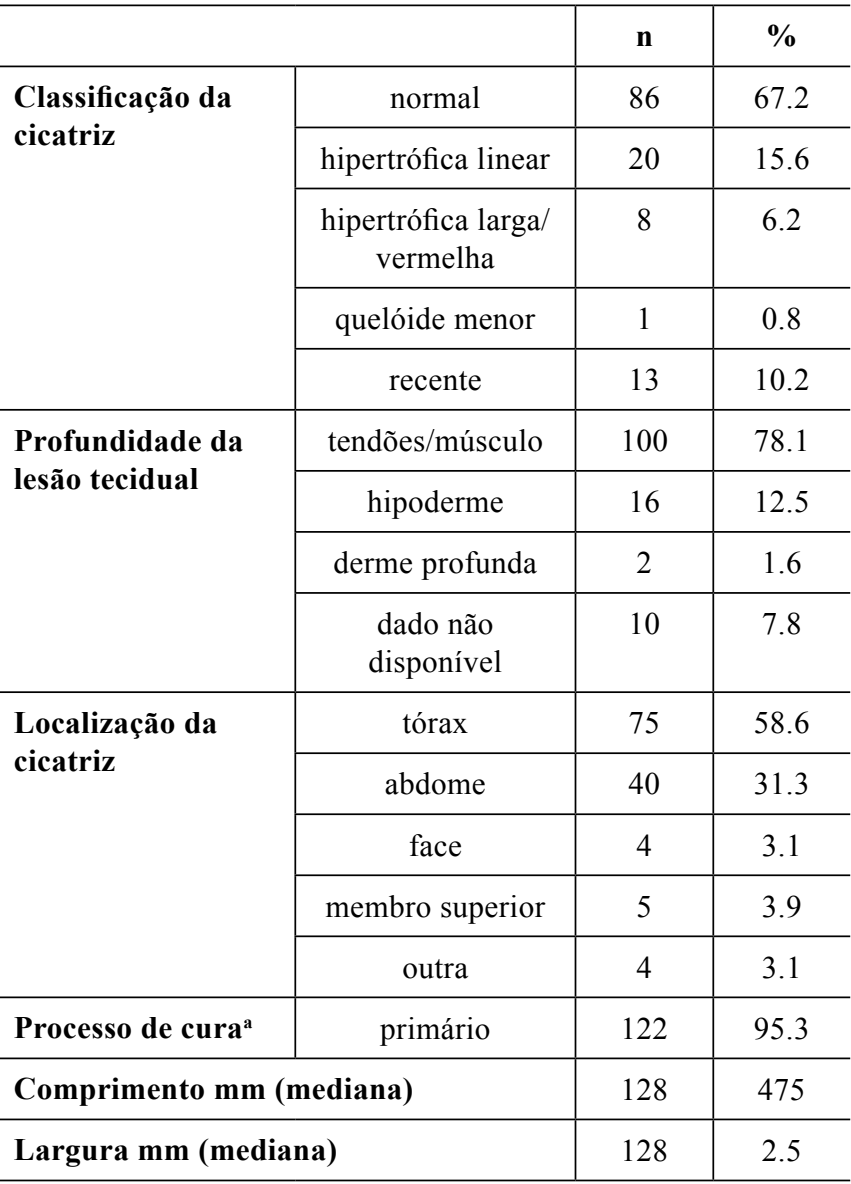

${ }^{a}$ primário ou secundário

Tabela 4. Classificação inicial da cicatriz.

\begin{tabular}{l|l|c|c}
\hline \multicolumn{1}{l|}{ Sintomas e sinais pré-tratamento } & n & \% \\
\hline \multirow{4}{*}{ Sintoma } & prurido & 46 & 66.7 \\
\cline { 2 - 4 } & fisgada & 26 & 37.7 \\
\cline { 2 - 4 } & dor & 22 & 31.9 \\
\cline { 2 - 4 } & sensibilidade/tato & 9 & 13 \\
\cline { 2 - 4 } & pontada & 9 & 13 \\
\cline { 2 - 4 } & repuxamento & 19 & 27.5 \\
\cline { 2 - 4 } & pelo menos um presente & 13 & 18.8 \\
\hline \multirow{4}{*}{ Sinal } & eritema & 60 & 87 \\
\cline { 2 - 4 } & relevo & 33 & 47.8 \\
\cline { 2 - 4 } & endurecimento & 28 & 40.6 \\
\cline { 2 - 4 } & alargamento & 25 & 36.2 \\
\cline { 2 - 4 } & pelo menos um presente & 5 & 7.2 \\
\hline
\end{tabular}




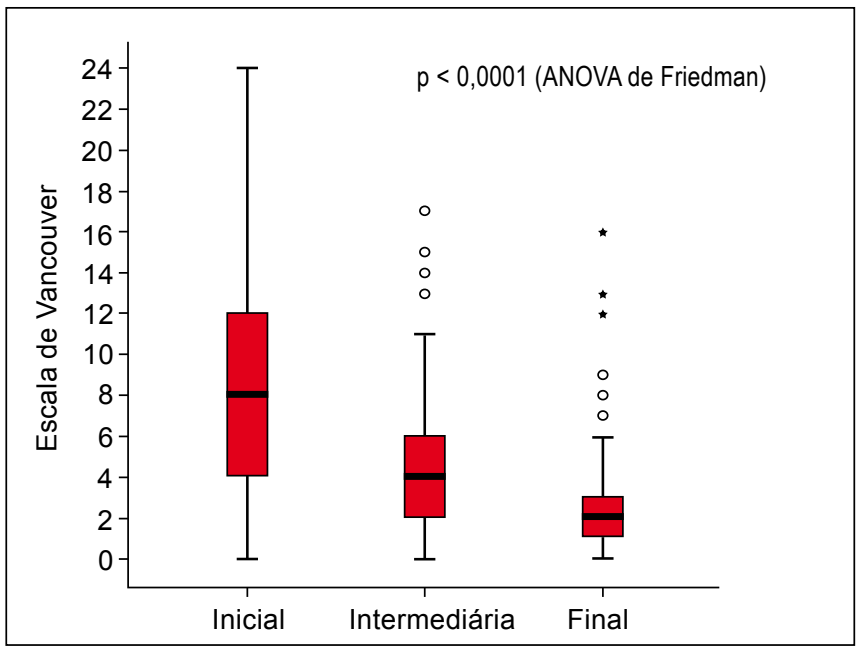

Figura 1 - Avaliação de Vancouver. Somatório da pontuação obtida com a escala de Vancouver nas consultas inicial, intermediária e final. Os resultados estão representados pela mediana, valores interquartis e valores mínimo e máximo para cada variável. Teste ANOVA de Friedman, $p<0,0001$.
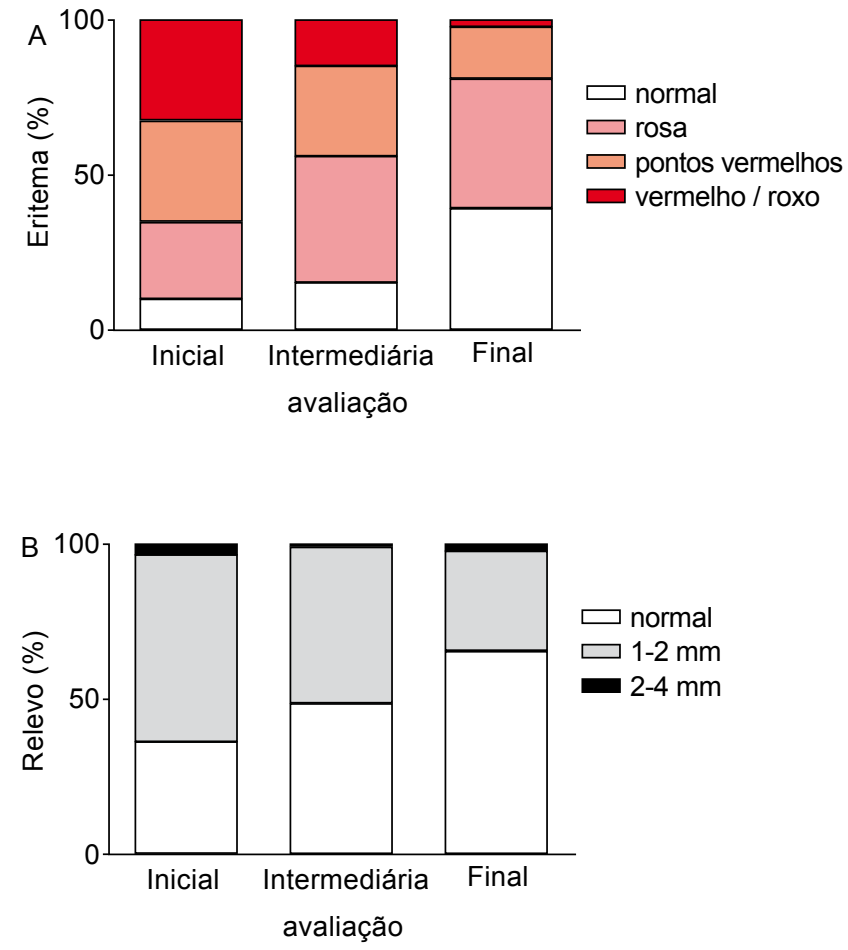

Figura 2 - Evolução percentual de sinais e sintomas. A: Percentual de eritema, classificado de acordo com a escala de Vancouver, nas visitas inicial, intermediária e final. B: Percentual de relevo, classificado de acordo com a escala de Vancouver, nas visitas inicial, intermediária e final.

\section{DISCUSSÃO}

O processo de cicatrização é dinâmico, dividido classicamente em três fases que se sobrepõem: inflamação, proliferação e remodelação. É após a $2^{\text {a }}$ semana da injúria que se inicia o remodelamento do colágeno, com constante síntese e degradação. $\mathrm{O}$ aumento do número de ligações cruzadas determina o progressivo fortalecimento da cicatriz, que continuará se remodelando por um longo período, mas sem nunca alcançar a resistência normal da pele. A alteração básica que ocorre, tanto na cicatriz hipertrófica como no quelóide, é uma proliferação continuada de tecido fibroso. A distinção entre as duas entidades é a tendência que o quelóide possui em se projetar além dos limites originais da lesão.

Incisões cirúrgicas são geralmente feridas limpas, possuindo as melhores condições para uma evolução favorável. Todo cirurgião, de qualquer especialidade, deve orientar seu paciente quanto aos cuidados preventivos para assegurar a melhor qualidade da cicatriz final. Após a retirada das últimas suturas (normalmente até o final da $2^{\mathrm{a}}$ semana pós-operatória), medidas gerais devem ser adotadas para acelerar a evolução da cicatriz. Além de beneficiá-la diretamente, é importante envolver o paciente, conscientizando-o de seu papel nos cuidados da sua cicatriz, a saber: massagens diárias com óleo mineral (para hidratar a cicatriz e manipular a rede de colágeno em formação); proteção contra irradiação solar; aplicação constante de silicone gel por pelo menos três meses.

$\mathrm{O}$ uso de gel de silicone em cicatrizes data do começo da década de 1980, inicialmente indicado no tratamento de cicatrizes extensas pós-queimadura. Desde então, um grande número de trabalhos, e dezenas de diferentes fórmulas do silicone gel, vêm sendo apresentados, atestando os benefícios deste produto na prevenção de cicatrizes hipertróficas e quelóides. Entretanto, o mecanismo exato de ação ainda é debatido. A hipótese mais aceita é que o curativo oclusivo - obtido com as diferentes apresentações do silicone gel - estimula os queratinócitos à maior secreção de fatores de crescimento localmente, influenciando consequentemente a regulação dos fibroblastos. Acredita-se também que a maior hidratação - pelo fator de oclusão - diminui a permeabilidade capilar, os mediadores inflamatórios e mitogênicos, e a síntese de colágeno.

Diversos produtos à base de silicone gel estão disponíveis no mercado, e a literatura revela que não há diferença alguma entre eles, no que tange a melhora na qualidade da cicatriz. São todos biocompatíveis, transparentes e inodoros, e podem ser usados na população pediátrica. $\mathrm{O}$ gel forma uma camada semi-oclusiva à água e permeável às trocas gasosas. A sua apresentação varia, porém, podendo ser em fita, em gel coesivo, e na forma de gel líquido de secagem rápida. Uma fórmula em spray está sendo desenvolvida, permitindo uma aplicação rápida em extensas áreas. Apesar das semelhanças, existem vantagens na aplicabilidade quando estes produtos são comparados entre si. Por ser 
um gel de rápida secagem (menos de 1 minuto), o produto usado neste estudo não é pegajoso, permitindo usar roupas diretamente em contato com a cicatriz. Também é possível passar outras substâncias sobre o filme de silicone gel, como protetor solar ou cremes de maquiagem. Por fim, ao contrário de fitas de silicone gel, é possível usar o gel líquido de secagem rápida em áreas expostas, como a face, sem a necessidade de fitas aderentes para manter o produto em contato com a pele.

Neste trabalho, após acompanhamento de 69 pacientes por seis meses, observamos melhora progressiva no aspecto da cicatriz, avaliado com a escala de Vancouver. Em geral, a melhora nos sintomas foi observada, sem diferenças adicionais, entre os períodos inicial e intermediário, como em prurido e sensibilidade (toda melhora foi observada no $1^{\circ}$ período, de três meses). Mas nos sinais avaliados, como eritema, relevo e flexibilidade, foi detectada melhora adicional entre a visita intermediária e final.

O grupo pesquisado foi representativo de uma população típica miscigenada (portanto mais sujeita às cicatrizes menos favoráveis), dando maior importância ao estudo, uma vez que a literatura somente relata trabalhos realizados em pacientes de cor clara. Entretanto, no presente estudo, foi decidido não tratar apenas parte da cicatriz (mantendo a outra não tratada para controle), nem tampouco houve grupo paralelo sem tratamento (grupo controle), o que permitiria uma análise comparativa entre o benefício do produto e pacientes sem nenhum tratamento. Tal comparação foi feita empiricamente, com a experiência clínica dos pesquisadores.

Recomenda-se iniciar o uso do silicone gel tão logo as últimas suturas tenham sido retiradas. Não se deve aplicar o produto em feridas abertas, nem em pacientes com história de alergia ao silicone. Idealmente, deve-se deixar o produto em contato com a cicatriz 24 h/dia, o que requer sua aplicação uma ou duas vezes ao dia, dependendo do hábito do paciente em banhar-se. O consenso da literatura é de uso contínuo do gel de silicone por três meses; não havendo melhora, deve-se estender o tempo de aplicação por mais três meses.

\section{CONCLUSÃO}

Em cicatrizes cutâneas de cirurgias plásticas, tratadas com silicone gel de rápida secagem, o acompanhamento clínico de 3-6 meses revelou significativa melhora nos seguintes parâmetros: prurido e "repuxamento", endurecimento, elevação e vermelhidão. Uma comparação empírica com pacientes que não usam o silicone gel revela que estes benefícios são percebidos já nas primeiras semanas de uso. A tolerabilidade do produto tem sido excelente, com raros casos de irritação cutânea. O gel de silicone de rápida secagem provou ter os mesmos benefícios dos outros produtos à base de silicone, porém com as vantagens de mais fácil aplicação. O trabalho conclui que o uso de silicone gel em cicatrizes pós-cirurgia plástica é seguro e eficiente na prevenção de cicatrizes de má qualidade.

\section{REFERÊNCIAS}

1. Pitanguy I, Brentano JM, Bos H, Salgado FS, Mazzarone F. Sistematização dos curativos e acompanhamento pós-operatório em cirurgia estética. Rev Bras Cir. 1988;78(1):67-78.

2. Fagundes FP, Lago EHJ, Lima BB, Carneiro SC. Cicatrização, cicatrizes e curativos. In: Ramos-E-Silva M, Ribeiro de Castro MC, eds. Fundamentos de dermatologia. Rio de Janeiro:Atheneu;2009. p.2077100.

3. Perkins K, Davey RB, Wallis KA. Silicone gel: a new treatment for burn scars and contractures. Burns Incl Therm Inj. 1983;9(3):201-4.

4. Mustoe TA, Cooter RD, Gold MH, Hobbs FD, Ramelet AA, Shakespeare $\mathrm{PG}$, et al. International clinical recommendations on scar management. Plast Reconstr Surg. 2002;110(2):560-71.

5. Berman B, Perez OA, Konda S, Kohut BE, Viera MH, Delgado S, et al. A review of the biologic effects, clinical efficacy, and safety of silicone elastomer sheeting for hypertrophic and keloid scar treatment and management. Dermatol Surg. 2007;33(11):1291-302.

6. Reish RG, Eriksson E. Scars: a review of emerging and currently available therapies. Plast Reconstr Surg. 2008;122(4):1068-78.

7. Mustoe TA. Evolution of silicone therapy and mechanism of action in scar management. Aesthetic Plast Surg. 2008;32(1):82-92.

8. Wolfram D, Tzankov A, Pulzl P, Piza-Katzer H. Hypertrophic scars and keloids: a review of their pathophysiology, risk factors, and therapeutic management. Dermatol Surg. 2009;35(2):171-81.

\section{Correspondência para:}

Henrique N. Radwanski

Rua Dona Mariana, 143 - Rio de Janeiro, RJ, Brasil - CEP 22280-020

E-mail: dr.henrique@pilos.com.br 